\section{Demonstration of normoblasts in tissue sections by means of an immunohistochemical technique for haemoglobin}

\author{
J CROCKER, ${ }^{*}$ OHB GYDE, $\dagger$ RACHEL \\ JENKINS* Departments of "Histopathology and \\ $\dagger$ Haematology, East Birmingham Hospital, Bordes- \\ ley Green East, Birmingham, B9 5ST
}

The morphological differentiation between normoblasts and lymphocytes in conventionally stained sections of bone marrow trephines may be difficult. ${ }^{1}$ This is unfortunate, especially since the distinction between the two cell types may be quintessential in cases of suspected chronic lymphocytic leukaemia, lymphocytic lymphoma, or other low grade nonHodgkin lymphomas. Guidelines to this problem include the careful observation of nuclear detailfor example, normoblasts have homogenous densely basophilic round nuclei with cytoplasm which shows "halo" artefact. Conversely, the nuclei of lymphocytes are of a finely granular chromatin pattern and the cells appear to possess no cytoplasm.' None the less, the differences between the cell types are subjective and appearances may be altered by fixation technique.

To enable the recognition of normoblasts in paraffin embedded sections of bone marrow trephines we have made use of the peroxidaseantiperoxidase (PAP) method using a primary antiserum against human haemoglobin. $\ddagger$

\section{Material and methods}

Twenty trephine biopsies, taken by means of the Menghini needle technique from the same number of patients, were studied. Specimens with normal histological appearances were selected. The cores were fixed in $10 \%$ glacial acetic acid in $10 \%$ formol-saline, which simultaneously decalcifies and obviates the use of trypsin before immunostaining. ${ }^{23}$ Two specimens (one of lymph node, the other of spleen) showing extramedullary haemopoiesis were also studied. The samples were embedded in paraffin wax; sections were cut at 2-3 $\mu \mathrm{m}$ thickness and then submitted to the immunoperoxidase procedure. After blocking of endogenous peroxidase by $0.1 \%$ hydrochloric acid in methanol and washing, the sections were covered by rabbit antihuman

Accepted for publication 17 July 1984

‡Mercia Brocades Ltd, Weybridge, Surrey, UK haemoglobin serum at a titre of $1 / 250-1 / 750$ for 30 $\mathrm{min}$ at room temperature. After another wash, swine antirabbit serum was applied at a titre of $1 / 50$ for $30 \mathrm{~min}$; this was followed by rabbit PAP complex after another wash. The peroxidase was shown by means of the 3,3'-diaminobenzidine (DAB) reaction, followed by counterstaining with Mayer's haemalum, dehydration, clearing, and mounting in synthetic medium.

The usual controls were performed: the optimum titre was used in the initial stage, together with substitution of normal rabbit serum for the first antiserum and omission of each of the other antisera in sequence. The response of each section to $\mathrm{DAB}$ alone was also tested. The reaction was also blocked by preadsorption of the antihaemoglobin serum with haemoglobin. $\S$

To confirm the localisation of haemoglobin in the sections, unstained preparations were examined in aqueous mountant using ultraviolet light at $415 \mathrm{~nm}$ (the "Soret band"). The image obtained was enhanced by means of a plumbicon tube placed over the microscope and linked to a television monitor. Marked fields, with positive cells, were then examined after staining for haemoglobin.

\section{Results}

In all specimens cells with morphologies which corresponded to those of normoblasts were stained intensely, the reaction either obscuring or appearing as a rim of activity around the nucleus (Fig. 1). Erythrocytes were much less strongly stained, and other cell types, including other red cell precursors and representatives of different cell series, were completely negative.

Normoblasts identified by absorption at $415 \mathrm{~nm}$ showed corresponding activity when immunostained for haemoglobin. The dark cytoplasmic rim observed by the former technique was much less intense than that resulting from the latter method (Fig. 2a and b). Mature erythrocytes, however, were much more readily shown by ultraviolet absorption than with the immunoperoxidase reaction.

All negative controls gave negative reactions and staining was abolished by preadsorption with haemoglobin.

\section{Discussion}

The distinction between normoblasts and lymphocytes in bone marrow trephines may sometimes be difficult. Certain features, such as nuclear chromatin pattern and the "halo" effect in normoblasts, may

§Sigma, Poole, Dorset, England 


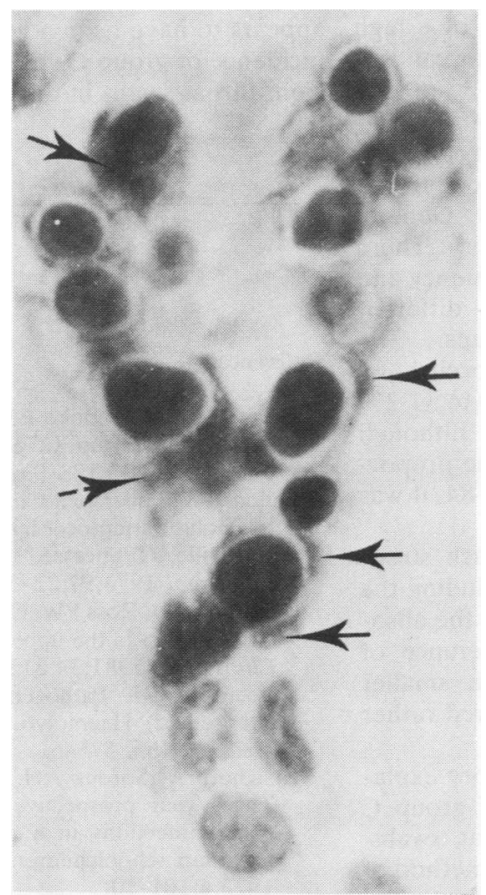

Fig. 1 Normoblasts, staining strongly in the cytoplasm for haemoglobin. Bone marrow trephine. Arrows indicate reaction product. PAP method for haemoglobin; haemalum counterstain $\times 1200$.

aid in this differentiation, ${ }^{1}$ but none the less difficulties may persist. This problem may be especially significant if diffuse infiltration of the marrow by, for example, malignant lymphocytes is suspected.

We suggest that immunoperoxidase staining of

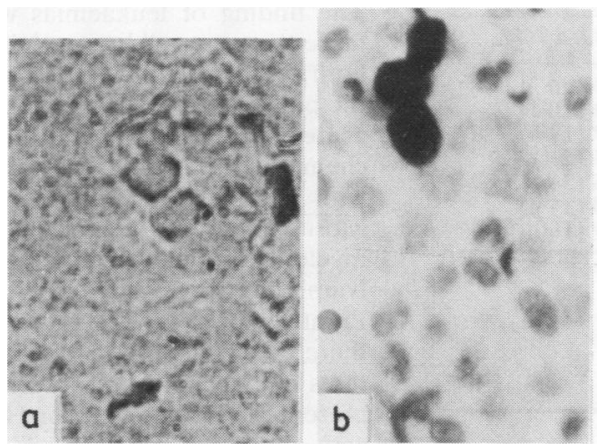

Fig. 2 (a) A small cluster of normoblasts shown in a paraffin embedded section, in the absence of staining, by virtue of absorption of ultraviolet light at $415 \mathrm{~nm}$ wavelength. A narrow but definite rim of absorption is seen in two cells as a rim of blackness around a white nucleus. (b) The same field, after immunostaining for haemoglobin, photographed in conventional light. There is intense staining, obscuring the nucleus in this case (b). $\times 400$. bone marrow for haemoglobin overcomes these problems. Trephines, fixed and decalcified in $10 \%$ acetic acid in $10 \%$ formol-saline, are ideally suited for immunohistochemical procedures ${ }^{2}$ and afford good morphological detail. The intensity of staining with the reaction is much greater than that of the effect resulting from absorption at $415 \mathrm{~nm}$. The relatively poor staining for haemoglobin seen in mature erythrocytes compared with the intense reaction found in normoblasts contrasts with the strength of absorption of ultraviolet light in the "Soret band." This may be explained by different membrane properties. Thus erythrocytes appear to be less damaged by tissue fixation and processing than normoblasts since, on the basis of absorption at $415 \mathrm{~nm}$, haemoglobin is clearly present in large amounts in erythrocytes in sections treated in this manner, and yet immunostaining is much reduced in them. This could result from less penetration after routine histological processing of the erythrocyte cell membrane than that of the normoblast by the antisera involved in this immunohistochemical procedure.

It has previously been shown that immunohistochemical staining for factor VIII related antigen provides $a^{\cdot}$ useful means of demonstrating extramedullary megakaryopoiesis ${ }^{4}$ and it appears that the current study is equally helpful in specimens of extramedullary erythropoiesis.

The preparations currently described would be well suited for the enumeration of normoblasts in automatic or semi-automatic image analysis systems, the immunoperoxidase reaction providing excellent tinctorial contrast. Such techniques would aid the recognition of early or established erythroid hyperplasia.

We are grateful to Mrs $\mathbf{R}$ Fry for her painstaking typing of the manuscript. This work was supported by a generous grant from the Research Committee of the West Midlands Region.

\section{References}

' Krause JR. Bone marrow biopsy. Edinburgh: Churchill Livingstone, 1981:33-4.

${ }^{2}$ Curran RC, Gregory J. The effects of fixation and processing on the immunohistochemical demonstration of immunoglobulin in paraffin sections of tonsil and bone marrow. J Clin Pathol 1980;33:1047-57

${ }^{3}$ Crocker J, Curran RC. Quantitative study of the immunoglobulin-containing cells in trephine samples of bone marrow. J Clin Pathol 1981;34:1080-2.

4 Crocker J, Smith PJ. The value of factor VIII related antigen as a means of demonstrating extramedullary megakaryopoiesis. $J$ Clin Pathol 1984;37:834-5.

Requests for reprints to: Dr J Crocker, Department of Histopathology, East Birmingham Hospital, Bordesley Green East, Birmingham B9 5ST, England. 